Royal Marsden NHS Foundation Trust, Surrey, UK

Correspondence to: Maggie Breen

Children's Unit, Downs Road, Sutton, Surrey SM2 5PT, UK; maggie.breen@rmh.nhs.uk

\title{
Parents of children who died from their brain tumours share common challenges during the neurological deterioration of their child, including balancing competing responsibilities and talking to their child about death
}

\section{Maggie Breen}

Commentary on: Zelcer S, Cataudella D, Cairney AE, et al. Palliative care of children with brain tumors: a parental perspective. Arch Pediatr Adolesc Med 2010;164:225-30.

Children with central nervous system (CNS) tumours have a higher mortality following their primary diagnosis than those with other childhood cancers (eg, leukaemia). Over the last decade, inadequate symptom relief and communication barriers between families and the healthcare team has been established and reported by parents caring for a child dying of cancer. A difference in the experience of end of life symptoms and parental experience by tumour type has been suggested in the literature. This paper explores the parental experience of caring for their children with terminal CNS tumours.

A qualitative design from the viewpoint of 25 bereaved parents (a self-selecting convenient sample) participated in three focus groups; all were 3-12 years post the death of their children. The semi structured questions aimed to measure the quality of life and healthcare satisfaction in children with terminal cancer.

Identified themes included the experience of parents living through the dying trajectory of their child, parental struggle in coping with the burden of care with competing parental responsibilities and barriers parents met over time to achieve their preferred place of care. Progressive disease affecting the child's perceived quality of life and a child helping their parents through the dying process was also highlighted.
Conclusions drawn increase awareness to the challenges families face and the demands on healthcare professionals to meet the needs of this patient population.

\section{Methodological shortcomings}

The study enriches understanding of the parents' experience and there is consistency in data explanation. However, no data was given on Canada's community palliative healthcare service and this may have had a greater influence on parental satisfaction and choice of preferred place of care than was reported.

No demographic data alluded to the multicultural, ethnicity or family composition of the 25 parents at the time of caring for their own children which may have influenced their care in decision making. Focus groups can build on the insight of others but are not independent opinion. The paper did not address how parents were assigned to the three focus groups or the purpose of videoing the group.

Within 1 year of their diagnosis, nine (over 52\%) children had died and it would have been 3-12 years (median 5.5 years) after the death of these children, so memory recall may have influenced parental responses. This paper 
did not record the ages of the 17 children who had died at home though Zelcer and colleagues discussed parental inability for their child to remain at home during their end of life care. Also for 11 (64\%) children who had died between ages 12 and 19 years, there was no evidence suggesting this group's involvement in their own care or preferred place of care.

Palliative care research is limited in relation to parental satisfaction of healthcare outcomes and may be bound up with ethical dilemmas with this area of research. Multicentre studies are usual within paediatric care; this is due to low numbers overall of children dying from advanced cancer.

\section{Limited clinical application}

Conclusions drawn cannot be generalised because the numbers are small, though they remain credible in qualitative research terms. Results do inform clinicians on the lived experience of a sample of parents in North America providing end of life care, but may not be applicable to an National Health Service (NHS) setting. Delivery of NHS services at the point of need is free and community nursing services are well established. Children's palliative care services as a subspecialty exists across the UK for those children dying from advanced cancer. Parental satisfaction of healthcare services provided and experienced by children and their families is currently being driven by the Department of Health care agenda. ${ }^{1}$
The children's hospice movement has evolved in the UK adding further choice in preferred place of care. My practice suggests children with CNS tumours and their families are more likely to engage with hospices, due to the progressive nature of their neurological deficits, for example sensory and intellectual deficits.

Parents want to take on increased responsibility for care of their sick child and this will not change; and professional support will continue to be required. I have experience of teenagers requesting to return to healthcare settings when actively dying. The reasons include the perceived burden of care their parents' experience, their adult size compromising care at home and the security of symptom management when professionals provide care.

Research exploring decision making processes by families would establish where the locus of control sits during end of life care to assist professionals in partnership working. Teenager's needs are thought to be distinct when receiving end of life care and further research is needed to establish this, involving teenagers themselves. Within palliative care settings qualitative research will continue to be the model of enquiry as 'meaning' is constructed not discovered.

Competing interests None.

\section{Reference}

1. Department of Health. Better Care: Better Lives. 2008. http:// www.dh.gov.uk/prod_consum_dh/groups/dh_digitalassets/ @dh/@en/documents/digitalasset/dh_083108.pdf (accessed 5th November 2009). 